\title{
Integrated omics analysis reveals sirtuin signaling is central to hepatic response to a high fructose diet
}

\author{
Laura A. Cox ${ }^{1,2,3,4^{*}}$, Jeannie Chan ${ }^{1,2}$, Prahlad Rao ${ }^{5}$, Zeeshan Hamid' ${ }^{1}$, Jeremy P. Glenn²,3, Avinash Jadhav ${ }^{1,2}$, \\ Vivek Das ${ }^{6}$, Genesio M. Karere ${ }^{1,2}$, Ellen Quillen ${ }^{1,2}$, Kylie Kavanagh $^{1,4}$ and Michael Olivier ${ }^{1,2}$
}

\begin{abstract}
Background: Dietary high fructose (HFr) is a known metabolic disruptor contributing to development of obesity and diabetes in Western societies. Initial molecular changes from exposure to HFr on liver metabolism may be essential to understand the perturbations leading to insulin resistance and abnormalities in lipid and carbohydrate metabolism. We studied vervet monkeys (Clorocebus aethiops sabaeus) fed a HFr $(n=5)$ or chow diet $(n=5)$ for 6 weeks, and obtained clinical measures of liver function, blood insulin, cholesterol and triglycerides. In addition, we performed untargeted global transcriptomics, proteomics, and metabolomics analyses on liver biopsies to determine the molecular impact of a HFr diet on coordinated pathways and networks that differed by diet.
\end{abstract}

Results: We show that integration of omics data sets improved statistical significance for some pathways and networks, and decreased significance for others, suggesting that multiple omics datasets enhance confidence in relevant pathway and network identification. Specifically, we found that sirtuin signaling and a peroxisome proliferator activated receptor alpha (PPARA) regulatory network were significantly altered in hepatic response to HFr. Integration of metabolomics and miRNAs data further strengthened our findings.

Conclusions: Our integrated analysis of three types of omics data with pathway and regulatory network analysis demonstrates the usefulness of this approach for discovery of molecular networks central to a biological response. In addition, metabolites aspartic acid and docosahexaenoic acid (DHA), protein ATG3, and genes ATG7, and HMGCS2 link sirtuin signaling and the PPARA network suggesting molecular mechanisms for altered hepatic gluconeogenesis from consumption of a HFr diet.

Keywords: High fructose diet, Integrated omics, Transcriptomics, Proteomics, Metabolomics, miRNA, Liver metabolism, Diet, Vervet, Pathway analysis

\section{Background}

Fructose intake in countries where people consume a Western diet has significantly increased over the past three decades, particularly through increased

*Correspondence: laurcox@wakehealth.edu

${ }^{1}$ Center for Precision Medicine, Department of Internal Medicine, Section on Molecular Medicine, Wake Forest School of Medicine, Medical Center Boulevard, NRC, G-floor, NC 27157 Winston-Salem, USA

Full list of author information is available at the end of the article consumption of sweetened beverages and foods containing high-fructose corn syrup. Fructose consumption comprises a significant proportion of energy intake in the American diet, and increased consumption coincides with increased prevalence of obesity over the past three decades [1]. Animal studies have shown that diets high in fructose consistently induce metabolic perturbations associated with metabolic syndrome and diabetes $[1,2]$. Altered metabolism in the liver has been implicated in multiple chronic metabolic diseases [3]. Several studies 
have investigated HFr diet challenges in humans $[4,5]$ and nonhuman primates (NHP) [6-9]. In cynomolgus monkeys (Macaca fascicularis), long-term exposure to high fructose (HFr) diets increased liver steatosis, with extent related to duration of fructose exposure [10], but questions remain about the initial molecular changes induced by high levels of fructose that result in long-term health complications.

The vervet monkey (Chlorocebus aethiops sabaeus) is a model for multiple human complex diseases including neurodegenerative disease [11], Alzheimer's disease [1215], diabetes, obesity and metabolism [16-18] and cardiovascular disease $[19,20]$ among others. Due to the high degree of genomic [21-23], physiologic and metabolic conservation between vervets and humans, results in vervets are translatable to understanding human health and disease. The ability to control environmental factors including diet and feasibility of collecting tissue biopsy samples from healthy animals, provide opportunities to investigate molecular mechanisms that are dysregulated prior to evidence of clinical disease. Studies in vervets related to metabolism have included diet interventions with variation in sources of protein, fat, and carbohydrate $[18,24,25]$; However, none of these studies in humans or NHP have used global untargeted omics approaches to identify potential molecular mechanisms underlying diet-induced changes in liver metabolism. In addition, no studies to date have generated an integrated comprehensive multi-omics dataset to better understand these molecular changes [26].

The goal of this study included examination of the impact of a short-term exposure to a HFr diet in the liver, a key organ mediating carbohydrate and lipid metabolism, by integrating high-throughput omics data and investigating the benefits of data integration across multiple omics domains. The short-term HFr diet exposure has no discernible impact on body weight, insulin sensitivity, blood pressure, or triglycerides. Total plasma cholesterol and measures of liver injury were greater in animals fed the HFr diet than controls. We examined whether early molecular alterations in liver can be detected prior to development of obesity and diabetes. We compared transcriptome, proteome, and metabolome data from livers of vervets challenged with a HFr diet for six weeks with those fed a chow diet. We demonstrate that the molecular information obtained from integrated analysis of multiomics datasets is more informative than analyses of any of the individual omics datasets. In addition, using this integrated omics approach, we identified sirtuin signaling and a peroxisome proliferator activated receptor alpha (PPARA) regulatory network as central to the hepatic short-term response to a HFr diet. Metabolites aspartic acid and DHA provide direct evidence on alterations in liver metabolism, and connect sirtuin signaling pathway and PPARA regulatory network, suggesting perturbations in these molecular mechanisms underlie altered hepatic gluconeogenesis in response to a short-term HFr diet.

\section{Results \\ Clinical and morphometric Data analysis}

Female age-matched vervet monkeys were fed a chow diet (controls, $n=5)$ or a HFr diet $(n=5)$ for six weeks. Morphometric measures at the end of challenge were not different between groups. Total plasma cholesterol was increased, and measures of liver injury, alanine aminotransferase, alkaline phosphatase, and gamma-glutamyl transpeptidase were increased in animals fed the HFr diet compared to controls (Table 1).

\section{Transcriptomics data analysis}

Comprehensive analysis of RNA expression has commonly been used to study the influence of genetic factors on phenotypic variation and is often used as a surrogate measure for functional alterations (potentially mediated by proteins or by alterations in metabolite levels). As a first step of our multi-omics characterization of liver biopsies from animals in this study, we performed RNA-Seq analyses on all samples. We identified 10,688 transcripts that passed quality filters. Of these, 467 were differentially expressed between liver samples from animals fed HFr and chow diets (unadjusted $\mathrm{p}<$ 0.05) (Additional file 1). Pathway enrichment analysis revealed that 51 pathways were different between $\mathrm{HFr}$ and chow including sirtuin signaling, remodeling of epithelial adherens junctions signaling, and necroptosis signaling ( $p$-value $<0.05$, Fig. 1 , Additional files 2 and $3)$. Regulatory network analysis resulted in 5 networks with predicted activation states. Four networks regulated by XBP1, PPARA, MITF, and KLF15 were predicted to activate downstream targets, and one network regulated by HDAC1 was predicted to inhibit downstream targets ( $p$-value < 0.05) (Fig. 2, Additional files 4 and 5). Regulators XBP1, PPARA, MITF, KLF15, and HDAC1 were expressed but not different between liver samples from HFr and chow-fed animals.

\section{Proteomics data analysis}

We analyzed liver-extracted proteins using standard mass spectrometry approaches as reported previously [27]. Overall, we were able to identify 2858 proteins across the 10 samples. Of these, 1594 proteins were identified in at least 3 of 5 samples from either the chow- or the HFr-fed animals, and 1172 proteins were identified in samples from at least 3 animals in each group. We included further analyses the 1172 


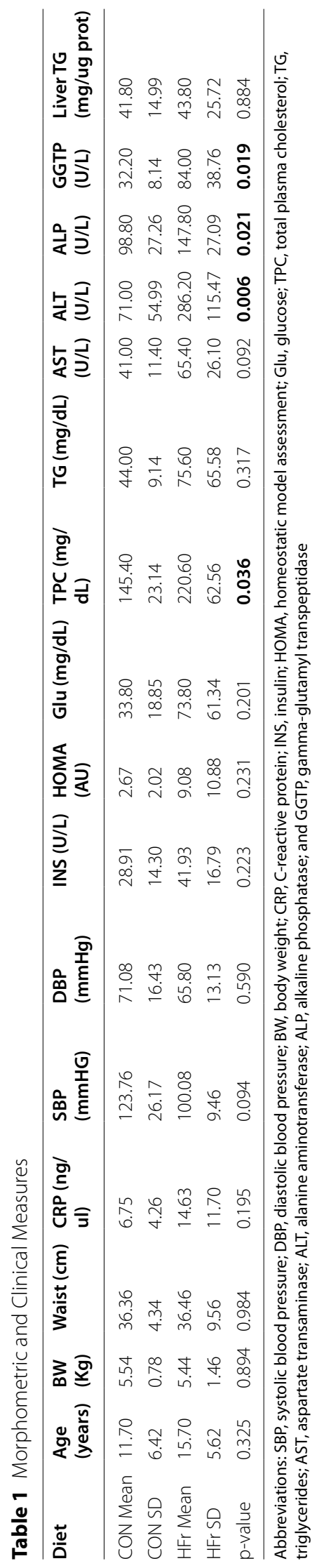




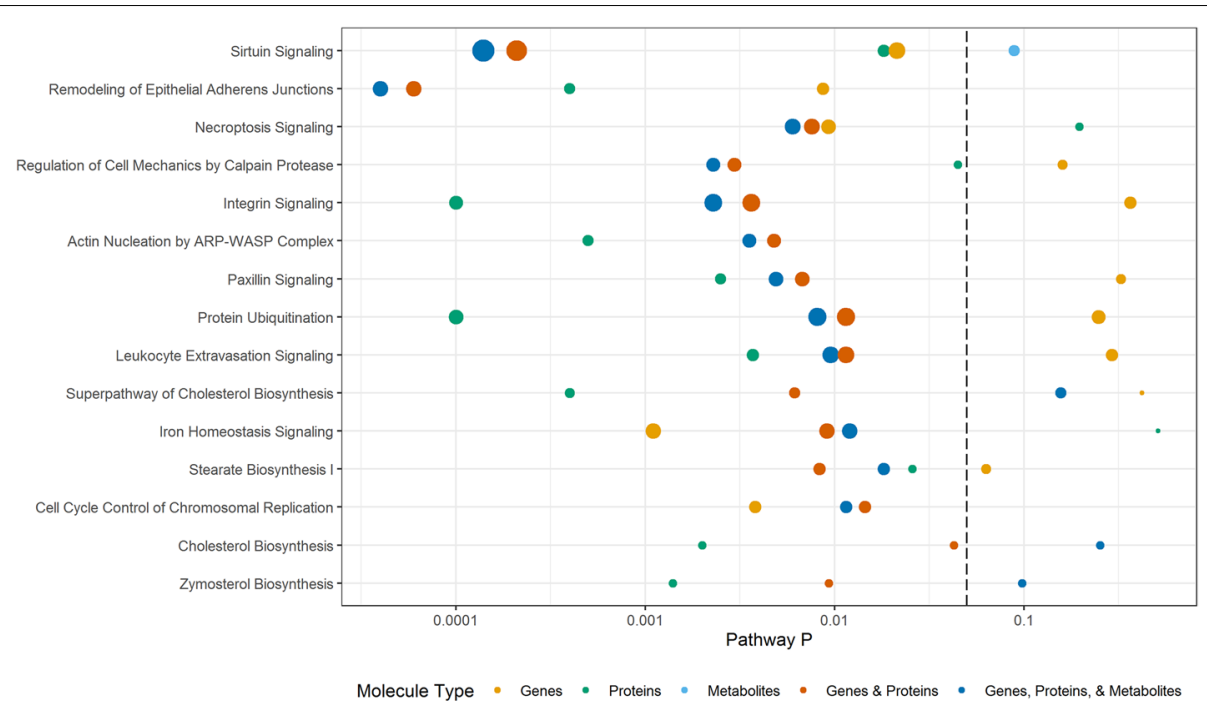

Molecules in Pathway $\bullet 5 \bullet 10 \bullet 15$

Fig. 1 Pathways for each omic data type and integrated omics data. P-values for each Ingenuity canonical pathway are plotted with the color indicating the type(s) of molecules included in the analysis and the dot size reflecting the number of molecules

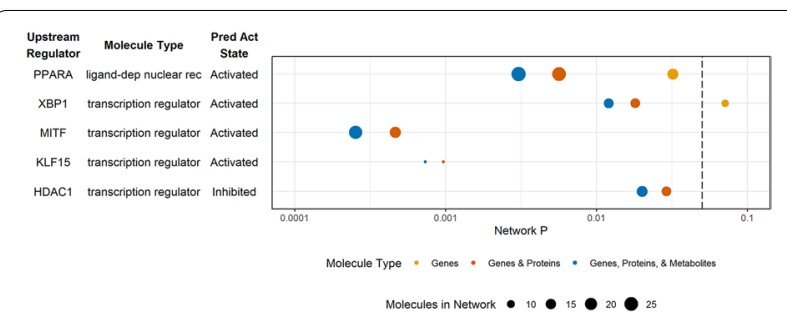

Fig. 2 Regulatory networks for each omic data type and integrated omics data. P-values for each network are plotted with the color indicating the type(s) of molecules included in the analysis and the dot size reflecting the number of molecules

proteins plus 70 proteins that passed quality filters for all samples in one group, but were not found in any of the samples of the other group. Of the combined 1242 proteins that passed these filters, 126 proteins were quantitatively different between liver samples from HFr- and chow-fed animals ( $p$-value $<0.05$ ) (Additional file 6). Pathway enrichment analysis revealed 58 pathways altered by HFr and included pathways that were also observed from the transcriptomic data, including sirtuin signaling, and remodeling of epithelial adherens junctions signaling ( $p$-value $<0.05$, Fig. 1 , Additional file 7). No regulatory networks were found with a predicted activation state (Fig. 2, Additional file 8). Network regulators XBP1, PPARA, MITF, KLF15, and HDAC1 were not detected in the proteomic analysis.

\section{Commonalities between gene and protein expression}

Comparison of gene and protein expression showed 320 molecules with greater expression and 263 with reduced expression that were common to both the transcriptomics and proteomics analyses in liver samples from animals fed a HFr diet compared to chow-fed animals. Comparison of statistically significant differentially expressed genes and proteins revealed only 2 shared molecules, SLCO1B1 and HTATIP2, with decreased abundance in livers from $\mathrm{HFr}$-fed animals compared to chow-fed animals (Fig. 3, Additional file 9).

\section{Metabolomics data analysis}

To examine whether we could expand on the molecular changes induced in the liver by HFr exposure that we uncovered by gene-centric analyses (transcriptomics, proteomics), we performed untargeted analysis of small molecule metabolites to analyze the metabolomic changes. Overall, we quantified 471 metabolites that passed quality filters. Of these, 18 showed significantly different abundances between liver samples from HFrand chow-fed animals ( $p$-value $<0.05$, Additional file 10 ). Pathway enrichment showed 25 pathways including aspartate biosynthesis. Sirtuin signaling was observed but not significant ( $p$-value $=0.089$, Fig. 1 and Additional file 11). All pathways identified in the enrichment analysis only contained one single metabolite per pathway, highlighting the limited annotation of metabolites in pathways and networks. No regulatory networks were 

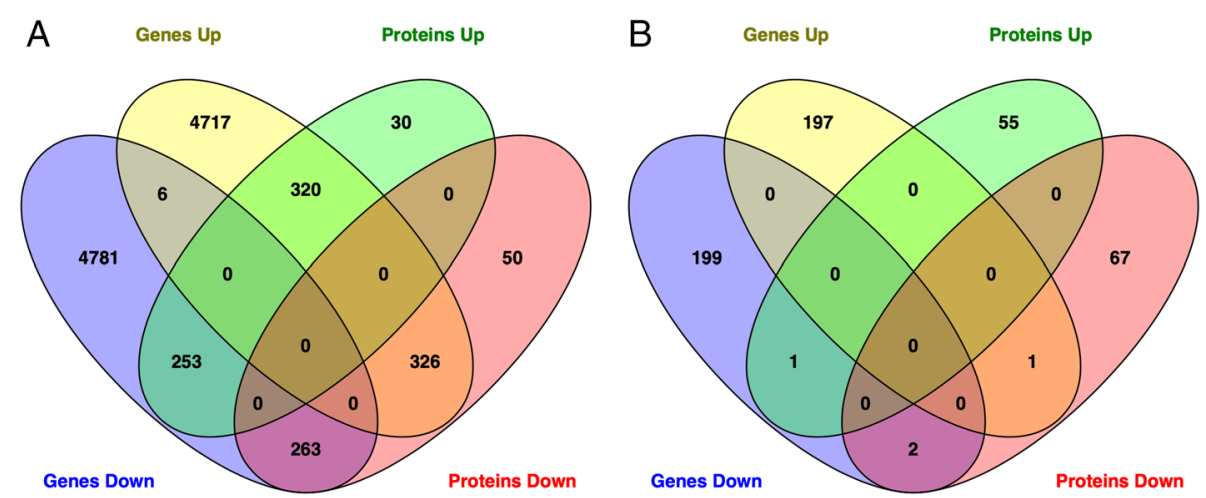

Fig. 3 Venn diagram showing (A) common expressed and (B) differentially expressed genes and proteins

found with a predicted activation state and $p$-value $<0.05$ (Fig. 2, Additional file 12).

\section{Integrated omics analysis}

Using the datasets described above, we further assessed whether combinations of omics datasets improved statistical confidence and significance in the network and pathway enrichment findings. First, we examined the combination of the gene expression and proteomics results. Integrated analysis of transcriptomic and proteomic data revealed 51 significantly enriched pathways ( $p$-value $<0.05)$. Statistical significance of sirtuin signaling, remodeling of epithelial adherens junctions, necroptosis signaling, and regulatory cell mechanics by calpain protease increased, and the number of molecules identified in each network increased with dataset integration. Interestingly, for sirtuin signaling, the number of genes and proteins was greater than the sum of genes and proteins from individual omic pathway analysis; this is due to our requirement for direct connections with addition of protein data to gene data connecting additional genes in the pathway. Significance of some pathways decreased, such as stearate biosynthesis, cell cycle control of chromosomal replication, and cholesterol biosynthesis (Fig. 2, Additional files 2 and 13). Integrated analysis showed 4 activated networks with predicted regulators PPARA, XBP1, MITF, and KLF15, and one inhibited network with predicted regulator HDAC1. Statistical significance increased and the number of molecules in the networks increased for the PPARA and XBP1 networks when compared to the analysis of the transcriptomic data alone (Fig. 3, Additional files 4 and 14).

Integration of the transcriptomics and proteomics data with metabolomics findings further enhanced the pathway enrichment and network analyses, and resulted in the identification of 43 significantly enriched pathways. The significance of several pathways, and the number of molecules identified in each pathway, increased even more compared to the gene-protein integrated pathways, including again sirtuin signaling, remodeling of epithelial adherens junctions, necroptosis signaling, and regulatory cell mechanics by calpain protease. Sirtuin signaling had the greatest significance and the greatest number of identified molecules with genes, proteins and metabolites. In addition, significance of other pathways such as cell cycle control of chromosomal replication, and cholesterol biosynthesis further decreased again when compared to the gene-protein integrated networks (Fig. 2, Additional files 2 and 15). Integrated network analysis was similar to pathway analysis with increased significance and molecule number compared to the gene-protein integrated networks, with the PPARA regulatory network (that included gene transcripts, proteins and metabolites) being the most significant (Fig. 3, Additional files 4 and 16). Of note, the protein FASN directly links regulatory networks PPARA, XBP1 and KLF15. In addition, overlapping molecules in networks link regulators PPARA and KLF15 with sirtuin signaling, including the protein ATG3, gene transcripts ATG7, and HMGCS2, and metabolites DHA and L-aspartic acid (Fig. 4).

\section{Integration of miRNA data}

In an effort to explore putative regulatory mechanisms underlying the pathway and network enrichment we describe above, we integrated analysis data from small RNA-Seq (which characterizes miRNAs) with the multiomics datasets described above. In our analysis, we identified 576 known miRNAs that passed quality filters. Of these, 22 were differentially expressed between liver samples from HFr- and chow-fed animals ( $p$-value < 0.05 , Additional file 17). Detailed miRNA - gene/protein pairing provided a list of 793 inverse pairs that included 17 differentially expressed miRNAs and 758 differentially abundant genes or proteins (Additional file 18). 


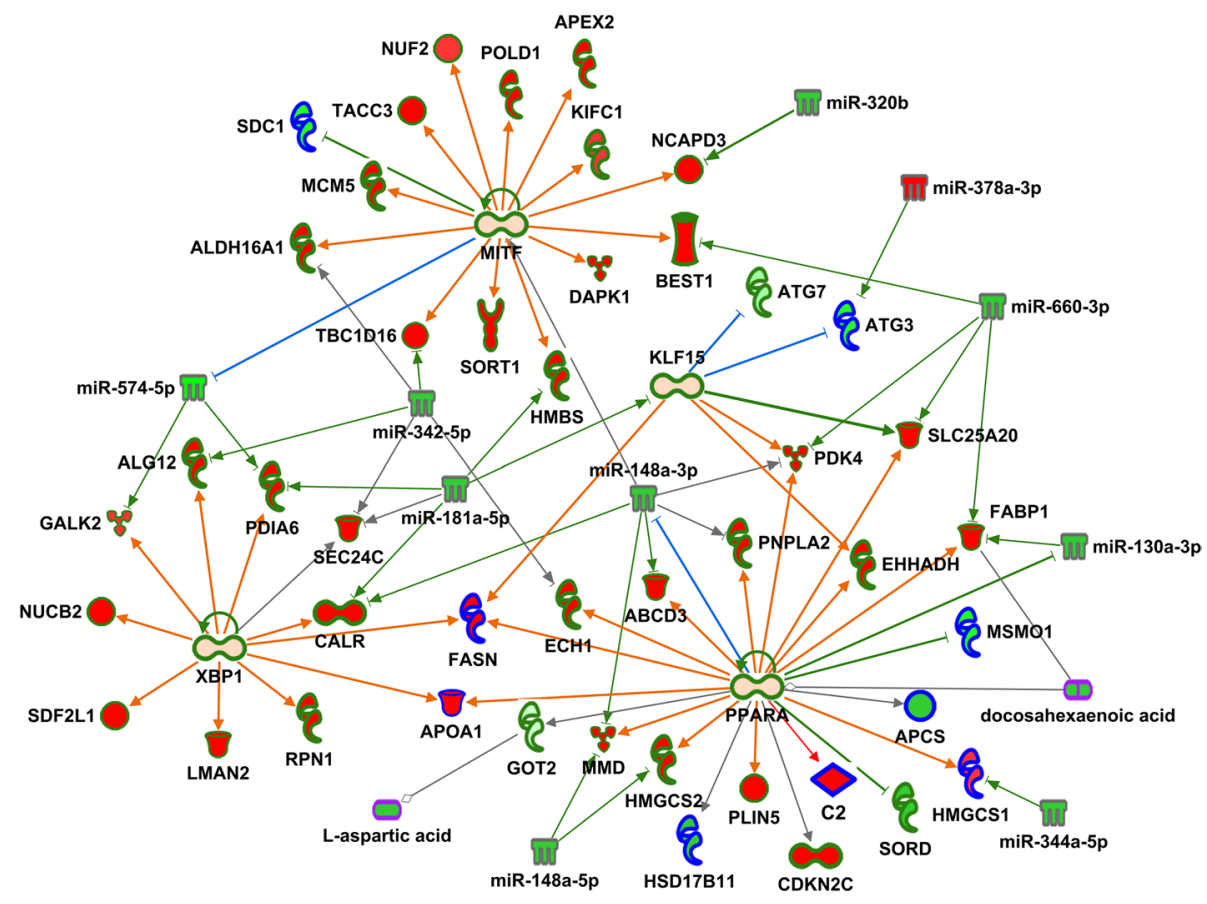

Fig. 4 Regulatory network up-regulated in HFr livers compared with chow. Red fill indicates increased abundance, green fill decreased abundance, light orange fill indicates predicted activation, green outline genes, blue outline proteins, gray outline miRNAs, purple outline metabolites, green lines indicate inhibition and red lines activation

Integration of miRNAs with pathways increased the number of molecules in remodeling of epithelial adherens junctions and necroptosis signaling, and the number of molecules increased for regulatory networks PPARA, XBP1, MITF and HDAC1 (Additional file 4). In addition, these regulatory networks were interconnected by miRNAs that target genes and proteins in multiple networks: miR-148-3p for PPARA, MITF, KLF15, and XBP1 network genes and proteins, miR-181a-5p for MITF, KLF15, and XBP2 network genes and proteins, miR 342-5p for MITF, XBP1 and PPARA network genes and proteins, and miR-574-5p for XBP1 and MITF network genes and proteins (Fig. 4). This integration suggests potential regulatory roles for these miRNAs in coordinating the molecular changes induced in the liver after exposure to a $\mathrm{HFr}$ diet, and emphasizes the complexity of miRNA interactions that may affect both transcript and protein levels.

\section{Genes and Proteins in Multi-Omic Networks with Associations to NASH- and NAFLD-Related Traits}

To examine the potential shared pathophysiological mechanisms induced by short term HFr diet exposure with long-term liver health outcomes associated with $\mathrm{HFr}$, we compared GWAS catalog variants and genes associated with nonalcoholic steatohepatitis (NASH)and nonalcoholic fatty liver disease (NAFLD)-related traits, including BMI, lipoproteins, obesity, diabetes, and insulin resistance, with the differentially expressed genes and proteins identified in our analysis of liver samples. The alignment of the datasets revealed 53 genes and proteins with one or more intergenic single nucleotide polymorphism (SNP) associated with one or more NASH/ NAFLD related trait(s) (Additional file 19). When we restricted the analysis only to genes and proteins in significantly enriched multi-omic pathways and networks, we identified 13 genes with GWAS SNPs, including FABP1 (associated with NAFLD) in PPARA and HDAC1 networks; GOT2 (associated with triglycerides and aspartate aminotransferase) in the sirtuin signaling pathway; and ATG7 (associated with fat body mass) in the sirtuin signaling pathway and KLF15 network (Table 2).

\section{Discussion}

The liver is central to metabolic regulation, and dysregulation of liver metabolism directly impacts gluconeogenesis and lipogenesis. Exposure to a HFr diet is known to increase the risk of dyslipidemia, insulin resistance, lipogenesis [28], levels of hepatic oxidative stress markers, and induce NASH and NAFLD [6]. Unlike glucose, fructose is absorbed in the intestine independently of energy or sodium exchange. When consumed in high amounts, fructose is transported to the liver via hepatic portal 
Table 2 Pathway and Network Genes and Proteins with GWAS SNPS

\begin{tabular}{|c|c|c|}
\hline Gene Symbol & Pathway or Network & Trait \\
\hline APOA1 & $\begin{array}{l}\text { HDAC1 Network } \\
\text { PPARA Network } \\
\text { XBP1 Network }\end{array}$ & Very low-density lipoprotein cholesterol \\
\hline ATG7 & $\begin{array}{l}\text { KLF15 Network } \\
\text { Sirtuin Signaling Pathway }\end{array}$ & Fat body mass \\
\hline CLIP1 & Remodeling of Epithelial Adherens Junctions & Body mass index \\
\hline FABP1 & $\begin{array}{l}\text { HDAC1 Network } \\
\text { PPARA Network }\end{array}$ & $\begin{array}{l}\text { Non-alcoholic fatty liver disease } \\
\text { Hepatic fibrosis }\end{array}$ \\
\hline GOT2 & Sirtuin Signaling Pathway & $\begin{array}{l}\text { Triglycerides } \\
\text { Aspartate aminotransferase }\end{array}$ \\
\hline MET & $\begin{array}{l}\text { MITF Network } \\
\text { Remodeling of Epithelial Adherens Junctions }\end{array}$ & Triglycerides \\
\hline MITF & MITF Network & $\begin{array}{l}\text { Low-density lipoprotein cholesterol } \\
\text { Triglycerides }\end{array}$ \\
\hline PNPLA2 & PPARA Network & Body fat distribution \\
\hline PPARA & PPARA Network & $\begin{array}{l}\text { Type II Diabetes } \\
\text { Total cholesterol } \\
\text { Low-density lipoprotein cholesterol } \\
\text { Triglycerides }\end{array}$ \\
\hline RAC1 & $\begin{array}{l}\text { Actin Nucleation by ARP-WASP Complex } \\
\text { Integrin Signaling } \\
\text { Leukocyte Extravasation Signaling } \\
\text { Paxillin Signaling }\end{array}$ & Low-density lipoprotein cholesterol \\
\hline RAP1GAP & Leukocyte Extravasation Signaling & Alkaline phosphatase \\
\hline SORT1 & MITF Network & $\begin{array}{l}\text { Type II Diabetes } \\
\text { Coronary artery disease } \\
\text { LDL cholesterol change }\end{array}$ \\
\hline TNFRSF11B & Necroptosis Signaling Pathway & Alkaline phosphatase \\
\hline
\end{tabular}

circulation and is preferentially converted to lipids. Fructose forms the building blocks of triglycerides [29], and triglycerides produced in the liver mostly are packaged into atherogenic very low-density lipoprotein particles [30]. Fructose in the liver can also serve as substrate for the gluconeogenesis pathway and increase circulating glucose levels [31], which, together with the increased triglyceride levels, decreases overall glycemic control. The specific contribution of hepatic steatosis to whole body insulin sensitivity and dyslipidemia [32-35] is particularly significant for individuals diagnosed with the metabolic syndrome. However, the underlying molecular networks that are dysregulated by a HFr diet and precede insulin resistance, NASH and NAFLD have not yet been identified, and the initial molecular abnormalities initiated by the exposure to fructose remain to be identified [6].

NHPs have been shown to be valuable models of dietinduced metabolic dysregulation due to extensive similarities with human metabolism [7]. The ability to carefully control diet exposure, and the physiological similarity to humans make NHP an ideal model to examine molecular tissue and organ changes in response to short- and long-term dietary challenges. We used a cohort of vervet monkeys (Clorocebus aethiops sabeus) fed an acute HFr $\operatorname{diet}(\mathrm{n}=5)$ or chow diet $(\mathrm{n}=5)$ for 6 weeks. Previous analyses showed changes in liver enzymes, total plasma cholesterol, and liver histology indicative of liver injury with periportal and inflammatory lesions in the HFr group [6], but no other clinically discernable abnormalities in body mass, or circulating glucose levels. In this study, we used global untargeted transcriptomics, proteomics, and metabolomics of liver biopsy samples to identify the acute early hepatic molecular and cellular response to a HFr diet, prior to onset of fat accumulation or systemic pathophysiological changes, to identify dysregulated molecular networks that potentially drive fat accumulation, and may be the initiating steps for subsequent longterm liver dysregulation. Pathway and network analyses were performed on individual datasets and integrated multi-omics datasets to determine whether there was a gain in our understanding of the molecular impact of a HFr diet with a combined approach compared to use of single or double omics datasets. Our analytical approach included prioritization of molecules by using pathway and network enrichment statistics, with the stringent requirement of direct connections among molecules, to 
improve statistical rigor for this study with small sample sizes (a common limitation of NHP studies).

We chose to use IPA to assess integrated omics effectiveness since it has tools for canonical pathway enrichment, and the underlying knowledgebase provides a means for regulatory network analysis at high resolution using transcripts, proteins, and metabolites, which is not yet feasible with other publicly available tools such as DAVID Bioinformatic Resources [36]. Our findings confirm previous papers indicating the need for better tools to perform integrated omic analyses [26]. In addition, it will be important to test strengths and limitations of multi-omics data integration with other tools when available.

In analyzing individual omics datasets, we identified a large number of statistically significant pathways for each data type, which is often the case for these types of data, making it a challenge to prioritize networks and distinguish likely true associations from spurious results. Integration of hepatic transcriptomic and proteomic data increased the significance of a number of pathways and networks, while decreasing the significance of other pathways, suggesting that truly associated pathways can be distinguished better with this approach. Interestingly, comparison of differentially expressed genes and proteins showed very little overlap: potentially due to the low correlation usually observed in expressed protein and transcript abundances. Most studies investigating proteome and transcriptome in the same model have noted this (e.g. [37]). However, integration of these datasets provided additional molecules with direct connections within a pathway or network, increasing the overall number of molecules, increasing the confidence in pathway or network prediction, and providing additional information about molecular functions. For some pathways and networks, additional differentially abundant molecules were added from the second omics dataset, creating new connections not evident in either of the individual omics datasets. Of note, proteins are often identified as molecules connecting separate regulatory networks and steps within signaling pathways, e.g. ATG3 in sirtuin signaling and FASN for the XBP1, PPARA and KLF15 networks.

Integration of transcriptomic and proteomic data increased the significance of the sirtuin signaling pathway, and revealed direct connections between sirtuin signaling and the four activated networks with predicted regulators PPARA, XBP1, MITF and KLF15. It is important to note that all of these genes were detected but not differentially expressed, but the encoded proteins were not detected. These results do not contradict the role of these proteins as central regulators since activity of all four depend on post-translational modifications, and the impact of these regulators may therefore not be mediated by changes in transcript or protein abundance [38-43].

Integration of metabolomic data with transcriptomic and proteomic datasets further improved significance of some pathways, with sirtuin signaling increasing in rank and statistics from being 7th for transcriptomics and 39 th for proteomics, to becoming 2nd for transcriptomics and proteomics, and 2nd overall with integration of all 3 datatypes. This pathway included the most molecules, including 4 metabolites. Other pathways decreased in significance and rank compared with the analysis of individual omics datasets. Addition of metabolites also provided more direct connections among regulatory networks, and connected the sirtuin signaling pathway with the PPARA network. Metabolites aspartic acid and DHA also indicated end-of-pathway directionality for the sirtuin signaling pathway and the PPARA network.

Finally, integration of miRNA data showed 19 of 22 differentially expressed miRNAs targeted genes and/or proteins in the four activated networks and sirtuin signaling pathway with inverse expression profiles. Our miRNA findings suggest that the initial hepatic response to shortterm exposure to a HFr diet is at least in part epigenetically regulated. Taken together, these results demonstrate that integration of transcriptomic, small transcriptomic, proteomic, and metabolomic data reveals pathways and networks central the HFr diet response in the liver, not seen by analysis of only one or two of these omic datasets.

Our results from these unique NHP biopsy samples reveal interesting novel molecular mechanisms regulating the initial hepatic response to HFr diet exposure in these animals. The sirtuin signaling pathway and networks regulated by PPARA, XBP1, MITF and KLF15 appear to be central to the HFr diet response. Both sirtuin signaling [44, 45] and PPARA [46] play important roles in the pathophysiology of NAFLD. For the sirtuin gene family, the majority of studies have focused on the role of SIRT1 in regulating both lipid and carbohydrate metabolism [47-49]. Interestingly, in our study, SIRT2 rather than SIRT1 was central to the initial hepatic response to a HFr diet. A recent study in male mice showed that SIRT2 functions as a negative regulator of NAFLD development and progression, with increased expression being protective when animals were fed a high-fat diet [50]. Our study in female NHPs showed higher SIRT2 expression in the HFr group compared with chow-fed animals, and lower expression of GOT2 and decreased abundance of aspartic acid [51], which is regulated by GOT2 [52, 53]. In mice, quantification of GOT2 protein expression by immunohistochemistry shows decreased abundance with NAFLD [54], supporting our preliminary findings. GOT2 and aspartic acid are at the end of the sirtuin pathway 
and indicative of altered gluconeogenesis and pathologies associated with NAFLD.

While the overall pathways identified in our analysis are supported by published evidence in other model organisms and related pathophysiologies, we also raise additional questions about previously under- or unappreciated regulatory networks. Our analysis suggests that the HFr diet exposure led to activation of the PPARA network, and downstream molecules GOT2 and aspartic acid showed decreased abundance. Studies of PPARA liver expression in mice with steatosis in response to a high-fat diet show sex-differences: PPARA expression is increased in male rats, and FASN, which is directly downstream of PPARA, is also increased. However, in female rats, FASN is increased but PPARA is not [55], suggesting that hepatic PPARA activation/inhibition of FASN may be sex-specific, and the potentially divergent expression patterns in our female NHP in response to the HFr diet may be specific to female animals.

As another example, our detailed multi-omics analysis also suggested that DHA, an omega-3 polyunsaturated fatty acid with anti-inflammatory functions [56], was lower in livers from animals fed a HFr diet than in livers from chow-fed animals. While no studies have reported changes in DHA in response to fructose, human studies examining dietary supplementation with DHA have suggested the beneficial effects of the increased level of DHA may include decreased incidence of NAFLD [57]. DHA is known to bind and activate PPARA [58] which may influence sirtuin signaling and the integrated regulatory network we discovered in our analysis. The decreased abundance of DHA, but with predicted activation of PPARA and activation of all but GOT2 downstream of PPARA, like aspartic acid, suggests differences between rodents and primates or sex-differences in these signaling networks, and may point to other mechanisms (apart from DHA) by which PPARA expression may be increased by HFr.

GWAS of genes and proteins in sirtuin signaling and the four activated networks we identified show a single gene, FABP1, that has been reported to be associated with alanine aminotransferase levels, a marker of liver disease [59]. Twelve additional genes were associated with lipoprotein-, insulin-, and BMI-related traits. Identification of SIRT2 and an integrated network of regulatory genes and proteins with altered abundance in livers from animals exposed to a HFr diet that are upstream of GOT2 and aspartic acid suggest that we have identified novel molecules and regulatory mechanisms that influence and potentially govern the initial hepatic response to short-term HFr diet exposure. Additional studies are required to validate our findings, and to explore potential targets by which these networks can be modulated to blunt the effects of fructose consumption on overall liver metabolism and function, preventing subsequent health complications known to occur with high intake levels.

\section{Conclusions}

We have demonstrated that integration of multiple omics datasets significantly improves prioritization of pathways and networks that influence hepatic response to a short-term HFr diet. Using this integrated approach, we identified sirtuin signaling and a large, integrated regulatory network, with molecules overlapping sirtuin signaling as a potential key modulator and regulator of hepatic metabolism in response to a HFr diet.

\section{Materials and methods \\ Animals and experimental design}

All experimental procedures involving vervet monkeys (Chlorocebus sabaeus) were approved and complied with the guidelines of the Institutional Animal Care and Use Committee of Wake Forest University Health Sciences, which is an AALAC accredited facility. The study was carried out in compliance with the ARRIVE guidelines. Procedures were performed by a veterinarian (KK), including liver biopsy as previously described [27]. Animals were provided non-steroidal anti-inflammatory and opioid analgesics during recovery as needed. Liver tissue was flash frozen in liquid nitrogen and stored at $-80^{\circ} \mathrm{C}$ until analysis. Animal housing, handling, diet compositions (chow and HFr) and caloric details are as described elsewhere [6]. Prior to the study, all animals were maintained on chow diet. For this study, 10 female vervet monkeys were fed with either chow $(n=5)$ or $\mathrm{HFr}$ $(n=5)$ diets for 6 weeks. Previous studies have shown sexspecific metabolic responses to a HFr diet [7]; for this reason, all animals included in the study were female. Animals were stratified to diet group balancing age and body weight (details are described in [6]. The data analysis team was blinded to the intervention stage of the trial (i.e., feeding and thus nutrient exposure); no data reported in the manuscript have a subjective element.

\section{Clinical measures}

Serum-based clinical measures, including total protein, albumin, globulin, albumin/globulin ratio, AST, ALT, ALK phosphatase, GGTP, total bilirubin, urea nitrogen, creatinine, BUN/creatinine ratio, phosphorus, glucose, calcium, magnesium, sodium, potassium, $\mathrm{Na} / \mathrm{K}$ ratio, chloride, cholesterol, triglycerides, amylase, lipase, $\mathrm{CPK}$, and hematological parameters including WBC, $\mathrm{RBC}$, hemoglobin, hematocrit, $\mathrm{MCV}, \mathrm{MCH}, \mathrm{MCHC}$, blood parasites, platelet count, platelet, EST, neutrophils, bands, lymphocytes, monocytes, eosinophils and 
basophil data were obtained from ANTECH Diagnostics (800-872-1001, NC, USA).

\section{Transcriptomics: RNA Seq}

\section{RNA Extractions and Sequencing}

Total RNA was extracted from vervet monkey livers using the Zymo Direct-zol ${ }^{\mathrm{TM}}$ kit (Zymo Research) and each sample was subsequently quantified by Qubit assay (Thermo Fisher). RNA-Seq libraries were prepared from $500 \mathrm{ng}$ of total RNA according to the Illumina TruSeq stranded mRNA protocol (Illumina), which specifically retains polyadenylated mRNAs by the oligo dT coated magnetic beads. Sequencing library concentrations were quantified using the KAPA library quantification kit (Kapa Biosystems). Clusters were generated by cBot (Illumina), and $2 \times 100$ base paired-end sequencing libraries were sequenced using the Illumina HiSeq 2500 with v3 sequencing reagents (Illumina).

Data Analysis: Raw sequences were de-multiplexed using the Illumina pipeline CASAVA v1.8. The FastQC and FASTX toolkit were used for QC. Sequence reads with Phred scores $\geq$ Q30 were retained. Reads aligned against the vervet reference genome (ChlSab1.1) were annotated using the Ensembl release 93 gene model. Abundance analysis was performed using our established RNA-Seq workflow in Partek Flow, which allowed calculation of transcript-level expression of a gene's isoforms for alternative spliced transcripts [60, 61]. Transcript abundances were quantified in Flow (Partek) using an expectation-maximization algorithm similar to the reported [62] which quantifies isoform expression levels across the whole genome at the same time and normalizes by transcript length to account for the transcript fragmentation step in RNA-Seq. Transcripts without read counts across all samples were filtered out, and then normalized by the trimmed mean of $M$ values method [Robinson MD and Oshlack A. Genome Biol. 11:R25, 2010] Differentially expressed genes were identified by 2 -sided $\mathrm{t}$-test assuming equal variance (unadjusted $\mathrm{p}<0.05$ ). Gene expression data were deposited in the National Center for Biotechnology Information's Gene Expression Omnibus (GEO; http://www.ncbi.nlm.nih.gov/geo/) GEO Series accession number GSE176576.

\section{Transcriptomics: small RNA Seq}

\section{Sequencing}

RNA extracted for RNA Seq was also used for small RNA Seq. Small RNA Seq methods are described in [63]. Briefly, small RNA sequencing libraries were prepared using the Illumina TruSeq Small RNA Sample Prep Kit and were pooled after cDNA synthesis. cDNA libraries were clustered using an Illumina Cluster Station and sequenced with an Illumina GAIIx sequencer. Raw sequence reads were obtained using Illumina's Pipeline v1.5. Extracted sequence reads were normalized, annotated and abundance determined using mirDeep2 [64].

\section{Transcriptomics: Data Analysis}

Transcripts without read counts across all samples were filtered out, and then normalized by the trimmed mean of $M$ values method. Differentially expressed genes were identified by 2 -sided $\mathrm{t}$-test assuming equal variance (unadjusted $\mathrm{p}<0.05$ ). Gene expression data were deposited in the National Center for Biotechnology Information's Gene Expression Omnibus (GEO; http://www.ncbi. nlm.nih.gov/geo/) - GEO accession number GSE178269.

\section{Proteomics}

Proteomics data were generated by liquid chromatography-coupled tandem mass spectrometry using a Thermo Scientific Orbitrap Elite mass spectrometer. Details of sample preparation, mass spectral analysis, and data analysis using a proteogenomics approach in Morpheus. The proteogenomics approach in our Morpheus analysis, used the vervet RNA-Seq data as a reference database. The match is made with the gene symbol/annotation provided from the RNA-Seq data, which for most protein assignments, eliminates one protein matching multiple gene symbols. Details of this approach were described previously [27]. Only unique assignments were included in the pathway analysis, and peptides matching to gene families were not considered for the pathway and network enrichment.

\section{Proteomics: Data Analysis}

For each animal, peptide spectrum intensities reported in Morpheus were summed across occurrences (i.e., across multiple transcript matches) based on Gene IDs. Proteins identified and quantified in at least 3 animals per group (HFr and chow) retained for downstream analysis. Additionally, proteins that were quantified in all samples of one group but not in any of the samples of other group were also retained for subsequent analyses. Intensity values were log transformed, and missing data (at most 2 animals per group) were imputed using the NAguideR tool with the impseq approach (sequential imputation) separately for the two experimental groups (HFr or chow). Differentially abundant proteins were identified by 2 -sided $\mathrm{t}$-test assuming equal variance (unadjusted $\mathrm{p}$ $<0.05)$.

\section{Comparison of gene and protein abundance}

Gene lists (Additional file 1) and protein lists (Additional file 6) were uploaded into Venny and Venn diagrams were generated showing commonly expressed and 
differentially expressed genes and proteins [65]. Ratios of HFr to chow were used to determine directionality.

\section{Metabolomics GC-TOFMS Analysis}

Liver metabolites were analyzed with chemical derivatization following previously published protocols [66, 67]. Extracted samples were spiked with two internal standard solutions $(10 \mu \mathrm{L}$ of L-2-chlorophenylalanine in water, $0.3 \mathrm{mg} / \mathrm{mL} ; 10 \mu \mathrm{L}$ of heptadecanoic acid in methanol, $1 \mathrm{mg} / \mathrm{mL}$ ), mixed, and extracted with $300 \mu \mathrm{L}$ of metha$\mathrm{nol} /$ chloroform (3:1). After centrifugation at $12000 \mathrm{~g}$ for $10 \mathrm{~min}$, an aliquot of the $300-\mu \mathrm{L}$ supernatant was transferred to a glass sampling vial to vacuum-dry at room temperature. The residue was derivatized using a twostep procedure. First, $80 \mu \mathrm{L}$ of methoxyamine $(15 \mathrm{mg} / \mathrm{mL}$ in pyridine) was added to the vial and kept at $30{ }^{\circ} \mathrm{C}$ for $90 \mathrm{~min}$, followed by $80 \mu \mathrm{L}$ of BSTFA ( $1 \%$ TMCS) at $70{ }^{\circ} \mathrm{C}$ for $60 \mathrm{~min}$.

Each $1-\mu \mathrm{L}$ aliquot of the derivatized solution was injected in splitless mode into an Agilent $6890 \mathrm{~N}$ gas chromatograph coupled with a Pegasus HT time-of-flight mass spectrometer (Leco Corporation, St. Joseph, MI). The CRC and control samples were run in the order of "control-CRC-control", alternately, to minimize systematic analytical deviations. Separation was achieved on a DB- $5 \mathrm{~ms}$ capillary column $(30 \mathrm{~m} \times 250 \mu \mathrm{m}$ i.d., $0.25-\mu \mathrm{m}$ film thickness; (5\%-phenyl)-methylpolysiloxane bonded and cross-linked; Agilent J\&W Scientific, Folsom, CA), with helium as the carrier gas at a constant flow rate of $1.0 \mathrm{~mL} / \mathrm{min}$. The temperature of injection, transfer interface, and ion source was set to 270,260 , and $200{ }^{\circ} \mathrm{C}$, respectively. The GC temperature programming was set to $2 \mathrm{~min}$ isothermal heating at $80^{\circ} \mathrm{C}$, followed by $10{ }^{\circ} \mathrm{C} /$ min oven temperature ramps to $180{ }^{\circ} \mathrm{C}, 5{ }^{\circ} \mathrm{C} / \mathrm{min}$ to $240{ }^{\circ} \mathrm{C}$, and $25^{\circ} \mathrm{C} / \mathrm{min}$ to $290{ }^{\circ} \mathrm{C}$, and a final 9 min maintenance at $290{ }^{\circ} \mathrm{C}$. Electron impact ionization $(70 \mathrm{eV})$ at full scan mode $(m / z 30-600)$ was used, with an acquisition rate of 20 spectra/s in the TOFMS setting.

\section{GC-TOFMS Data Analysis}

The acquired MS files from GC-TOFMS analysis were exported in NetCDF format by ChromaTOF software (v3.30, Leco Co., CA). CDF files were extracted using custom scripts (revised Matlab toolbox hierarchical multivariate curve resolution (H-MCR), developed [68, 69] in the MATLAB 7.0 (The MathWorks, Inc.) for data pretreatment procedures such as baseline correction, denoising, smoothing, alignment, time-window splitting, and multivariate curve resolution (based on multivariate curve resolution algorithm) [68]. The resulting data set includes sample information, peak retention time and peak intensities. Compound identification was performed by comparing the mass fragments with National Institute of Standards and Technology (NIST) 05 Standard mass spectral databases in NIST MS search 2.0 (NIST, Gaithersburg, MD) software with a similarity of more than $70 \%$ and finally verified by available reference compounds. Differentially abundant metabolites for all MS analyses were identified by 2 -sided $t$-test assuming equal variance (unadjusted $\mathrm{p}<0.05$ ).

\section{D GC-ToF-MS Analysis}

Gas chromatography-mass spectrometry was performed as described [70]. Metabolite extracts were dried under vacuum in cold, and were then sequentially derivatized with methoxyamine hydrochloride (MeOX) and $\mathrm{N}$-methyl- $\mathrm{N}$-trimethylsilyl-trifluoroacetamide (MSTFA) [70]. One microliter of the derivatized sample was injected in splitless mode using an autosampler (VCTS, Gerstel $^{\mathrm{TM}}$, Linthicum, MD, USA) into a GC-MS system consisting of an Agilent $\odot 7890$ B gas chromatograph (Agilent Technologies, Palo Alto, CA, USA) with Pegasus ${ }^{\circledR}$ 4D ToF-MS instrument (LECO Corp., San Jose, CA, USA) equipped with an electron impact (EI) ionization source. Injection of the sample was performed at $250{ }^{\circ} \mathrm{C}$ with helium as a carrier gas and flow set to $2 \mathrm{~mL} \mathrm{~min}^{-1}$. GC was performed using a primary $\mathrm{Rxi}^{\circledR}{ }^{\circledR}-5 \mathrm{Sil}$ MS capillary column (Cat. No. 13623-6850, Restek, Bellefonte, PA, USA) $(30 \mathrm{~m} \times 0.25 \mathrm{~mm} \times 0.25 \mu \mathrm{m})$ and a secondary Rtx ${ }^{\circledR}$-17Sil capillary column (Cat. No. 40201-6850, Restek, Bellefonte, PA, USA). The temperature program started isothermal at $70{ }^{\circ} \mathrm{C}$ for $1 \mathrm{~min}$ followed by a $6{ }^{\circ} \mathrm{C}$ $\mathrm{min}^{-1}$ ramp to $310{ }^{\circ} \mathrm{C}$ and a final $11 \mathrm{~min}$ hold at $310^{\circ} \mathrm{C}$. The system was then temperature-equilibrated at $70{ }^{\circ} \mathrm{C}$ for $5 \mathrm{~min}$ before the next injection. Mass spectra were collected at $20 \mathrm{scans} / \mathrm{s}$ with a range of $\mathrm{m} / z$ 40-600. The transfer line and the ion source temperatures were set to $280{ }^{\circ} \mathrm{C}$. QC standards were injected at scheduled intervals for tentative identification and monitoring shifts in retention indices (RI).

\section{D GC-ToF-MS Data Analysis}

The GC-MS data were pre-processed, cleaned, aligned, and processed using ChromaToF version 4.50.8.0 (LECO Corp., Michigan, USA) following settings from [71]. Briefly described settings viz. S/N: 5; peak width: 0.15 , base line offset: $1 ; \mathrm{m} / \mathrm{z}$ range: $50-800$. The aligned data were also deconvoluted using Automated Mass Spectral Deconvolution and Identification System (AMDIS, NIST, USA) interface to match against the freely available MSRI spectral libraries of the Golm Metabolome Database available from Max-Planck-Institute for Plant Physiology, Golm, Germany (http://csbdb.mpimp-golm. mpg.de/csbdb/gmd/gmd.html) by matching the mass spectra and RI [72]. Metabolites were identified by 
comparing fragmentation patterns available in both the Golm database as well as NIST Mass Spectral Reference Library (NIST11/2011; National Institute of Standards and Technology, USA) library. Peak finding and quantification of selective ion traces were accomplished using AMDIS software. Base peak areas of the mass fragments $(\mathrm{m} / \mathrm{z})$ were normalized using median normalization and $\log _{2}$ transformation. Peak areas were normalized by dividing each peak area value by the area of the internal standard for a specific sample, and were further median normalized.

\section{Liquid Chromatography-Time of Flight Mass Spectrometry (LC-TOFMS)}

Plasma samples were processed as reported before [73]. A volume of $100 \mu \mathrm{L}$ supernatant was mixed with $400 \mu \mathrm{L}$ of a mixture of methanol and acetonitrile (5:3). Liver tissue homogenate was added to $500 \mu \mathrm{L}$ of a chloroform, methanol, and water mixture $(1: 2: 1, \mathrm{v} / \mathrm{v} / \mathrm{v})$. These samples were then mixed and centrifuged at 13,000 rpm for $10 \mathrm{~min}$ at $4{ }^{\circ} \mathrm{C}$. A $150 \mu \mathrm{L}$ aliquot of supernatant was transferred to a sampling vial. The deposit was rehomogenized with $500 \mu \mathrm{L}$ methanol followed by a second centrifugation. Another $150 \mu \mathrm{L}$ supernatant was added to the same vial for drying and then reconstituted in $500 \mu \mathrm{L}$ of ACN: $\mathrm{H} 2 \mathrm{O}(6: 4, v / v)$ before separation.

An Agilent HPLC 1200 system equipped with a binary solvent delivery manager and a sample manager (Agilent Corporation, Santa Clara, CA, USA) was used with chromatographic separations performed on a $4.6 \times 150$ mm $5 \mu \mathrm{m}$ Agilent ZORBAX Eclipse XDB-C18 chromatography column. The LC elution conditions are optimized as follows: isocratic at $1 \% \mathrm{~B}(0-0.5 \mathrm{~min})$, linear gradient from 1 to $20 \% \mathrm{~B}(0.5-9.0 \mathrm{~min}), 20-75 \% \mathrm{~B}$ (9.0-15.0 $\mathrm{min}), 75-100 \% \mathrm{~B}(15.0-18.0 \mathrm{~min})$, isocratic at $100 \% \mathrm{~B}(18-19.5 \mathrm{~min})$; linear gradient from 100 to $1 \% \mathrm{~B}$ $(19.5-20.0 \mathrm{~min})$ and isocratic at $1 \% \mathrm{~B}(20.0-25.0 \mathrm{~min})$. For positive ion mode $(\mathrm{ESI}+)$ where $\mathrm{A}=$ water with $0.1 \%$ formic acid and $\mathrm{B}=$ acetonitrile with $0.1 \%$ formic acid, while $\mathrm{A}=$ water and $\mathrm{B}=$ acetonitrile for negative ion mode (ESI-). The column was maintained at $30{ }^{\circ} \mathrm{C}$ as a $5 \mu \mathrm{L}$ aliquot of sample is injected. Mass spectrometry is performed using an Agilent model 6220 MSD TOF mass spectrometer equipped with a dual sprayer electrospray ionization source (Agilent Corporation, Santa Clara, CA, USA). The TOF mass spectrometer was operated with the following optimized conditions: [1] ES+ mode, capillary voltage $3500 \mathrm{~V}$, nebulizer $45 \mathrm{psig}$, drying gas temperature $325^{\circ} \mathrm{C}$, drying gas flow $11 \mathrm{~L} / \mathrm{min}$, and [2] ES- mode, similar conditions as ES + mode except the capillary voltage was adjusted to $3000 \mathrm{~V}$. During metabolite profiling experiments, both plot and centroid data are acquired for each sample from 50 to $1,000 \mathrm{Da}$ over a $25 \mathrm{~min}$ analysis time. Data generated from LC-TOFMS were centroided, deisotoped, and converted to mzData $\mathrm{xml}$ files using the MassHunter Qualitative Analysis Program (vB.03.01) (Agilent). Following conversion, xml files are analyzed using the open source XCMS package (v1.16.3) (http:// metlin.scripps.edu), which runs in the statistical package R (v.2.9.2) (http://www.r-project.org), to pick, align, and quantify features (chromatographic events corresponding to specific $\mathrm{m} / \mathrm{z}$ values and elution times). The software is used with default settings as described (http://metlin. scripps.edu) except for xset $(\mathrm{bw}=5)$ and rector (plottype $=$ " $\mathrm{m}$ ", family $=$ "s"). The created .tsv file is opened using Excel software and saved as .xls file. Compound identification was performed by comparing the accurate mass and retention time with reference standards available in our laboratory, or comparing the accurate mass with online database such as the Human Metabolome Database (HMDB).

Metabolomic LC/GC-TOFMS data was analyzed using principle component analysis (PCA) and OPLS analysis between groups. The differential metabolites were selected when they meet the requirements of variable importance in the projection (VIP) $>1$ in OPLS model and $\mathrm{p}<0.05$ from student $t$-test. The corresponding fold change shows how these selected differential metabolites varied from control. Final data analysis between control HFr-diet groups for each metabolite was conducted using independent $t$-test analysis with a $p<0.05$ significance threshold.

\section{Pathway and Network Analyses}

For individual omic datasets, all quality molecules for the dataset were uploaded to Ingenuity Pathway Analysis (IPA; QIAGEN). Unique gene symbols were used for genes and proteins, which are conserved between human and vervet. Pathway and network enrichment analyses used differentially abundant molecules and the IPA Knowledge Base, and requiring direct connections based on experimental evidence among differentially abundant molecules. Regulatory network prediction required previous experimental validation of direct connections in liver or liver cells. Right-tailed Fisher's exact test was used to calculate enrichment of differentially expressed genes in pathways, $\mathrm{p}<0.01[61]$.

\section{Integrated Omic Analyses}

Multi-omic data analysis combined the total gene, protein, and/or metabolite lists for all molecules that passed quality filters as appropriate for the data type. Lists included molecule ID, direction of change, fold change, and p-value. Pathway and network enrichment used the same parameters and statistical tests as for individual 
omic datasets, requiring experimentally validated direct connections for differentially abundant molecules.

IPA calculation of p-values for a given pathway, function, or network takes into account the following: (A) The number of associated molecules in the pathway, function, or network; (B) The number of eligible analysis-ready molecules in the dataset with that pathway, function, or network annotation; (C) The total number of molecules associated with that annotation and are included in the selected reference set; (D) The total number of molecules in the reference set; and (E) The total number of analysis-ready molecules that did not match that annotation. When molecules from multiple datasets are combined, the total number of molecules in the dataset increases, i.e., the denominator in the formula. If for example, a pathway is significant for transcript data, but no proteins or metabolites are directly connected to any of the genes in the pathway, then the p-value for that pathway will increase for the combined dataset compared to the transcriptome only dataset.

\section{miRNA - Gene/Protein pairing}

Current pathway and network enrichment tools in IPA do not provide the means to filter direct connections based on inverse abundance between a miRNA and its target. In order to integrate our miRNA data, we performed miRNA - gene pairing in IPA for our miRNA, gene and protein datasets, requiring opposite expression for experimentally validated or highly predicted interactions (e.g., HFr miRNA up-regulated and HFr gene down-regulated compared with chow). Using the gene and protein IDs in this list, we merged it with the list of genes and proteins in all significantly enriched pathways and networks. This analysis does not provide the means to statistically evaluate the significance of miRNA addition to a given pathway or network; however, this approach provides evidence of an epigenetic component of the liver response to HFr diet.

\section{Identification of pathway and network genes previously associated with NASH/NAFLD related traits}

The following search terms, with all variation of names in the GWAS catalog, were used to query the current GWAS catalog [74]: alkaline phosphatase, aspartate aminotransferase, body mass index, body weight, fasting blood glucose, fasting blood insulin, fat body mass, fatty acid, glucose, HbA1c, HDL cholesterol change, insulin, insulin resistance, insulin sensitivity, LDL cholesterol change, lipid, liver fat, liver disease biomarker, liver fibrosis, low density lipoprotein cholesterol, non-alcoholic fatty liver disease, non-alcoholic steatohepatitis, obesity, omega-3 polyunsaturated fatty acid, omega- 6 polyunsaturated fatty acid, total cholesterol, triglyceride, type II diabetes mellitus, very low density lipoprotein cholesterol. Genes with associations, based on the GWAS catalog, to any of these traits were compared to the list of all differentially expressed miRNAs, genes and proteins from our transcriptomic and proteomic datasets, and compared with the genes in proteins in multi-omic significant networks and pathways.

\begin{abstract}
Abbreviations
HFr: high fructose; PPARA: peroxisome proliferator activated receptor alpha; DHA: docosahexaenoic acid; NHP: nonhuman primates; NASH: nonalcoholic steatohepatitis; NAFLD: nonalcoholic fatty liver disease; GEO: Gene Expression Omnibus; H-MCR: hierarchical multivariate curve resolution; MeOX: methoxyamine hydrochloride; MSTFA: N-methyl-N-trimethylsilyl-trifluoroacetamide; El: electron impact; RI: retention indices; AMDIS: Automated Mass Spectral Deconvolution and Identification System; NIST: National Institute of Standards and Technology; LC-TOFMS: Liquid Chromatography-Time of Flight Mass Spectrometry; HMDB: Human Metabolome Database; PCA: principle component analysis; VIP: variable importance in the projection; IPA: Ingenuity Pathway Analysis.
\end{abstract}

\section{Supplementary Information}

The online version contains supplementary material available at https://doi. org/10.1186/s12864-021-08166-0.

Additional file 1. Gene List: Genes passing quality filters with ratios and p-values for HFr versus CON.

Additional file 2. Pathway Summary for genes, proteins, metabolites, combined genes and proteins, combined genes, proteins, and metabolites, and combined genes, proteins, metabolites, and miRNAs.

Additional file 3. Gene Pathways: Enrichment analysis of genes with p-value $<0.05$.

Additional file 4. Network Summary for genes, proteins, metabolites, combined genes and proteins, combined genes, proteins, and metabolites, and combined genes, proteins, metabolites, and miRNAs.

Additional file 5. Gene Networks: Enrichment analysis of genes with p-value $<0.05$.

Additional file 6. Protein List: Proteins passing quality filters with ratios and $\mathrm{p}$-values for HFr versus CON.

Additional file 7. Protein Pathways: Enrichment analysis of proteins with $\mathrm{p}$-value $<0.05$

Additional file 8. Protein Networks: Enrichment analysis of proteins with p-value $<0.05$.

Additional file 9. Common Genes and Proteins: List of common genes and proteins from Venney merge for all genes and proteins passing quality filters and for all differentially expressed genes and proteins.

Additional file 10. Metabolite List: Metabolites passing quality filters with ratios and $p$-values for $\mathrm{HFr}$ versus CON.

Additional file 11. Metabolite Pathways: Enrichment analysis of metabolites with $p$-value $<0.05$.

Additional file 12. Metabolite Networks: Enrichment analysis of metabolites with p-value \& 0.05 .

Additional file 13. Gene \& Protein Pathways: Enrichment analysis combining genes and proteins with $p$-value $<0.05$.

Additional file 14. Gene \& Protein Networks: Enrichment analysis combining genes and proteins with $p$-value $<0.05$.

Additional file 15. Gene, Protein, and Metabolite Pathways: Enrichment analysis combining genes, proteins, and metabolites with $p$-value $<0.05$ 
Additional file 16. Gene, Protein, and Metabolite Networks.

Additional file 17. miRNAs passing quality filters and $p$-values $<0.05$ for HFr versus CON

Additional file 18. Gene-Protein with miRNA pairs: miRNA pairing with target genes and proteins either highly predicted or experimentally validated for differentially expressed miRNAs, genes and proteins for $\mathrm{HFr}$ versus CON ( $p$-value < 0.05).

Additional file 19. Differentially Expressed Gene and Protein: List of GWAS hits of differentially expressed genes and proteins for HFr versus CON.

\section{Acknowledgements}

We thank Biswapriya Misra for contributing to generation of metabolomics data.

\section{Authors' contributions}

LAC, KK, and MO conceived the project. JPG, AJ, PR, GMK, LAC, JC, ZH, EQ, VD, and $\mathrm{MO}$ contributed to data generation and analyses. All authors read and approved the final manuscript

\section{Funding}

The animal work was supported by grants to KK: UL1TR001420, P400D010965, and K01AG033641; a portion of the analytical work was supported by grants to EQ: K01 AG056663, and GMK: K01 HL130697.

\section{Availability of data and materials}

RNA Seq, proteomic, and metabolomic data are available in Additional files. Raw RNA Seq data are available through NCBI GEO Series accession number GSE176576 and small RNA Seq data are available through GEO accession number GSE178269.

To review GEO accession GSE178269 go to:

https://urldefense.com/v3/_https://www.ncbi.nlm.nih.gov/geo/query/acc. cgi?acc=GSE178269_;!!GA8Xfdg!hpk17gHazQbLJ2Ux3leSzs9VDjsSSqkQ9zsG AAIMuyNtd_NsH2pPRYGKi1hk9Jw\$.

The following secure token has been created to allow review of record GSE178269 while it remains in private status: yryzskgcjrqfxav.

To review GEO accession GSE176576 go to: https://urldefense.com/v3/ https://www.ncbi.nIm.nih.gov/geo/query/acc.cgi?acc=GSE176576_;!'GA8Xfd g!iPSaGb7UXXtZbc2VRI0Nj1 cw9VE7rn_cFK62irhMe4UbjQs4vcXTLI31ISgLr38\$. The following secure token has been created to allow review of record GSE178269 while it remains in private status: knsrqcaodtoxjgz.

\section{Declarations}

\section{Ethics approval and consent to participate}

The study was carried out in compliance with the ARRIVE guidelines. All experimental procedures involving vervet monkeys (Chlorocebus sabaeus) were approved and complied with the guidelines of the Institutional Animal Care and Use Committee of Wake Forest University Health Sciences, which is an AALAC accredited facility. Procedures were performed by a board-certified veterinarian employed by Wake Forest University Health Sciences.

\section{Consent for publication}

All authors have reviewed the manuscript and consent for publication.

\section{Competing interests}

VD currently is a Post-Doctoral researcher at NNRCSI; however, he did not receive any funding for this work.

\footnotetext{
Author details

${ }^{1}$ Center for Precision Medicine, Department of Internal Medicine, Section on Molecular Medicine, Wake Forest School of Medicine, Medical Center Boulevard, NRC, G-floor, NC 27157 Winston-Salem, USA. ²Department of Genetics, Texas Biomedical Research Institute, 78245 San Antonio, TX, USA. ${ }^{3}$ Southwest National Primate Research Center, Texas Biomedical Research Institute, 78245 San Antonio, TX, USA. ${ }^{4}$ Department of Pathology, Section on Comparative Medicine, Wake Forest School of Medicine, 27157 Winston-Salem,
}

NC, USA. ${ }^{5}$ University of Tennessee Health Science Center, TN, Memphis, USA. ${ }^{6}$ Novo Nordisk Research Center, Seattle, WA, USA.

Received: 29 June 2021 Accepted: 8 November 2021

Published online: 03 December 2021

\section{References}

1. Elliott SS, Keim NL, Stern JS, Teff K, Havel PJ. Fructose, weight gain, and the insulin resistance syndrome. Am J Clin Nutr. 2002;76(5):911-22.

2. Havel PJ. Dietary fructose: implications for dysregulation of energy homeostasis and lipid/carbohydrate metabolism. Nutr Rev. 2005;63(5):133-57.

3. Softic S, Gupta MK, Wang GX, Fujisaka S, O'Neill BT, Rao TN, et al. Divergent effects of glucose and fructose on hepatic lipogenesis and insulin signaling. J Clin Invest. 2017;127(11):4059-74.

4. Teff KL, Elliott SS, Tschop M, Kieffer TJ, Rader D, Heiman M, et al. Dietary fructose reduces circulating insulin and leptin, attenuates postprandial suppression of ghrelin, and increases triglycerides in women. J Clin Endocrinol Metab. 2004;89(6):2963-72.

5. Stanhope KL, Havel PJ. Endocrine and metabolic effects of consuming beverages sweetened with fructose, glucose, sucrose, or high-fructose corn syrup. Am J Clin Nutr. 2008;88(6):1733S-7S.

6. Kavanagh K, Wylie AT, Tucker KL, Hamp TJ, Gharaibeh RZ, Fodor AA, et al. Dietary fructose induces endotoxemia and hepatic injury in calorically controlled primates. Am J Clin Nutr. 2013;98(2):349-57.

7. Bremer AA, Stanhope KL, Graham JL, Cummings BP, Wang W, Saville $B R$, et al. Fructose-fed rhesus monkeys: a nonhuman primate model of insulin resistance, metabolic syndrome, and type 2 diabetes. Clin Transl Sci. 2011;4(4):243-52.

8. Blevins JE, Graham JL, Morton GJ, Bales KL, Schwartz MW, Baskin DG, et al. Chronic oxytocin administration inhibits food intake, increases energy expenditure, and produces weight loss in fructose-fed obese rhesus monkeys. Am J Physiol Regul Integr Comp Physiol. 2015;308(5):R431-8.

9. Suzuki M, Yamamoto D, Suzuki T, Fujii M, Suzuki N, Fujishiro M, et al. High fat and high fructose diet induced intracranial atherosclerosis and enhanced vasoconstrictor responses in non-human primate. Life Sci. 2006;80(3):200-4.

10. Cydylo MA, Davis AT, Kavanagh K. Fatty liver promotes fibrosis in monkeys consuming high fructose. Obesity (Silver Spring). 2017;25(2):290-3.

11. Frye BM, Craft S, Latimer CS, Keene CD, Montine TJ, Register TC, et al. Aging-related Alzheimer's disease-like neuropathology and functional decline in captive vervet monkeys (Chlorocebus aethiops sabaeus). Am J Primatol. 2021:e23260.

12. Chen JA, Fears SC, Jasinska AJ, Huang A, Al-Sharif NB, Scheibel KE, et al. Neurodegenerative disease biomarkers Abeta1-40, Abeta1-42, tau, and p-tau181 in the vervet monkey cerebrospinal fluid: Relation to normal aging, genetic influences, and cerebral amyloid angiopathy. Brain Behav. 2018;8(2):e00903.

13. Kalinin S, Willard SL, Shively CA, Kaplan JR, Register TC, Jorgensen MJ, et al. Development of amyloid burden in African Green monkeys. Neurobiology of aging. 2013;34(10):2361-9.

14. Latimer CS, Shively CA, Keene CD, Jorgensen MJ, Andrews RN, Register TC, et al. A nonhuman primate model of early Alzheimer's disease pathologic change: Implications for disease pathogenesis. Alzheimers Dement. 2018.

15. Postupna N, Latimer CS, Larson EB, Sherfield E, Paladin J, Shively CA, et al. Human striatal dopaminergic and regional serotonergic synaptic degeneration with lewy body disease and inheritance of APOE epsilon4. Am J Pathol. 2017;187(4):884-95.

16. Kavanagh K, Davis AT, Peters DE, LeGrand AC, Bharadwaj MS, Molina AJ. Regulators of mitochondrial quality control differ in subcutaneous fat of metabolically healthy and unhealthy obese monkeys. Obesity. 2017;25(4):689-96.

17. Kavanagh K, Fairbanks LA, Bailey JN, Jorgensen MJ, Wilson M, Zhang L, et al. Characterization and heritability of obesity and associated risk factors in vervet monkeys. Obesity. 2007;15(7):1666-74.

18. Wilson QN, Wells M, Davis AT, Sherrill C, Tsilimigras MCB, Jones RB, et al. Greater microbial translocation and vulnerability to metabolic disease in healthy aged female monkeys. Sci Rep. 2018;8(1):11373. 
19. Jorgensen MJ, Rudel LL, Nudy M, Kaplan JR, Clarkson TB, Pajewski NM, et al. 25(OH)D3 and cardiovascular risk factors in female nonhuman primates. J Womens Health (Larchmt). 2012;21(9):959-65.

20. Jorgensen MJ, Aycock ST, Clarkson TB, Kaplan JR. Effects of a Western-type diet on plasma lipids and other cardiometabolic risk factors in African green monkeys (Chlorocebus aethiops sabaeus). J Am Assoc Lab Anim Sci. 2013;52(4):448-53.

21. Jasinska AJ, Zelaya I, Service SK, Peterson CB, Cantor RM, Choi OW, et al. Genetic variation and gene expression across multiple tissues and developmental stages in a nonhuman primate. Nat Genet. 2017;49(12):1714-21.

22. Schmitt CA, Service SK, Jasinska AJ, Dyer TD, Jorgensen MJ, Cantor RM, et al. Obesity and obesogenic growth are both highly heritable and modified by diet in a nonhuman primate model, the African green monkey (Chlorocebus aethiops sabaeus). International journal of obesity (2005). 2018:42(4):765-74.

23. Warren WC, Jasinska AJ, Garcia-Perez R, Svardal H, Tomlinson C, Rocchi M, et al. The genome of the vervet (Chlorocebus aethiops sabaeus). Genome Res. 2015;25(12):1921-33.

24. Kavanagh K, Wylie AT, Chavanne TJ, Jorgensen MJ, Voruganti VS, Comuzzie AG, et al. Aging does not reduce heat shock protein 70 in the absence of chronic insulin resistance. J Gerontol A Biol Sci Med Sci. 2012;67(10):1014-21.

25. Voruganti VS, Jorgensen MJ, Kaplan JR, Kavanagh K, Rudel LL, Temel $R$, et al. Significant genotype by diet $(G \times D)$ interaction effects on cardiometabolic responses to a pedigree-wide, dietary challenge in vervet monkeys (Chlorocebus aethiops sabaeus). Am J Primatol. 2013;75(5):491-9.

26. Misra BB, Langefeld CD, Olivier M, Cox LA. Integrated Omics: Tools, advances, and future approaches. J Mol Endocrinol. 2018.

27. Proffitt JM, Glenn J, Cesnik AJ, Jadhav A, Shortreed MR, Smith LM, et al. Proteomics in non-human primates: utilizing RNA-Seq data to improve protein identification by mass spectrometry in vervet monkeys. BMC Genomics. 2017;18(1):877.

28. Lecoultre V, Egli L, Carrel G, Theytaz F, Kreis R, Schneiter P, et al. Effects of fructose and glucose overfeeding on hepatic insulin sensitivity and intrahepatic lipids in healthy humans. Obesity (Silver Spring). 2013;21(4):782-5.

29. Cohen JC, Horton JD, Hobbs HH. Human fatty liver disease: old questions and new insights. Science. 2011;332(6037):1519-23.

30. Libby P, Ridker PM, Hansson GK. Progress and challenges in translating the biology of atherosclerosis. Nature. 2011;473(7347):317-25.

31. Rizkalla SW. Health implications of fructose consumption: A review of recent data. Nutr Metab (Lond). 2010;7:82.

32. Nguyen-Duy TB, Nichaman MZ, Church TS, Blair SN, Ross R. Visceral fat and liver fat are independent predictors of metabolic risk factors in men. Am J Physiol Endocrinol Metab. 2003;284(6):E1065-71.

33. Fabbrini E, Magkos F, Mohammed BS, Pietka T, Abumrad NA, Patterson BW, et al. Intrahepatic fat, not visceral fat, is linked with metabolic complications of obesity. Proc Natl Acad Sci U S A. 2009;106(36):15430-5.

34. Fabbrini E, Magkos F, Mohammed BS, Pietka T, Abumrad NA, Patterson BW, et al. Intrahepatic fat, not visceral fat, is linked with metabolic complications of obesity. P Natl Acad Sci USA. 2009;106(36):15430-5.

35. Nguyen-Duy TB, Nichaman MZ, Church TS, Blair SN, Ross R. Visceral fat and liver fat are independent predictors of metabolic risk factors in men. Am J Physiol-Endoc M. 2003;284(6):E1065-E71.

36. Jiao X, Sherman BT, Huang da W, Stephens R, Baseler MW, Lane HC, et al. DAVID-WS: a stateful web service to facilitate gene/protein list analysis. Bioinformatics. 2012;28(13):1805-6.

37. Bekker-Jensen DB, Kelstrup CD, Batth TS, Larsen SC, Haldrup C, Bramsen $\mathrm{JB}$, et al. An optimized shotgun strategy for the rapid generation of comprehensive human proteomes. Cell Syst. 2017:4(6):587-99 e4.

38. Sacco F, Humphrey SJ, Cox J, Mischnik M, Schulte A, Klabunde T, et al. Glucose-regulated and drug-perturbed phosphoproteome reveals molecular mechanisms controlling insulin secretion. Nat Commun. 2016;7:13250.

39. Wood P, Mulay V, Darabi M, Chan KC, Heeren J, Pol A, et al. Ras/mitogenactivated protein kinase (MAPK) signaling modulates protein stability and cell surface expression of scavenger receptor SR-BI. J Biol Chem. 2011;286(26):23077-92.
40. Wang FM, Chen YJ, Ouyang HJ. Regulation of unfolded protein response modulator XBP1s by acetylation and deacetylation. Biochem J. 2011:433(1):245-52.

41. Regazzetti C, Sormani L, Debayle D, Bernerd F, Tulic MK, De Donatis GM, et al. Melanocytes sense blue light and regulate pigmentation through opsin-3. J Invest Dermatol. 2018;138(1):171-8.

42. Wu M, Hemesath TJ, Takemoto CM, Horstmann MA, Wells AG, Price ER, et al. c-Kit triggers dual phosphorylations, which couple activation and degradation of the essential melanocyte factor Mi. Genes Dev. 2000;14(3):301-12.

43. Takeda K, Takemoto C, Kobayashi I, Watanabe A, Nobukuni Y, Fisher DE, et al. Ser298 of MITF, a mutation site in Waardenburg syndrome type 2, is a phosphorylation site with functional significance. Hum Mol Genet. 2000;9(1):125-32.

44. Ding S, Jiang J, Zhang G, Bu Y, Zhang G, Zhao X. Resveratrol and caloric restriction prevent hepatic steatosis by regulating SIRT1-autophagy pathway and alleviating endoplasmic reticulum stress in high-fat diet-fed rats. PLoS One. 2017:12(8):e0183541.

45. Nassir F, Ibdah JA. Sirtuins and nonalcoholic fatty liver disease. World J Gastroenterol. 2016;22(46):10084-92.

46. Regnier M, Polizzi A, Smati S, Lukowicz C, Fougerat A, Lippi Y, et al. Hepatocyte-specific deletion of Pparalpha promotes NAFLD in the context of obesity. Sci Rep. 2020;10(1):6489.

47. Colak Y, Yesil A, Mutlu HH, Caklili OT, Ulasoglu C, Senates E, et al. A potential treatment of non-alcoholic fatty liver disease with SIRT1 activators. J Gastrointestin Liver Dis. 2014;23(3):311-9.

48. Tobita T, Guzman-Lepe J, Takeishi K, Nakao T, Wang Y, Meng F, et al. SIRT1 disruption in human fetal hepatocytes leads to increased accumulation of glucose and lipids. PLoS One. 2016;11(2):e0149344.

49. Bruce KD, Szczepankiewicz D, Sihota KK, Ravindraanandan M, Thomas $\mathrm{H}$, Lillycrop KA, et al. Altered cellular redox status, sirtuin abundance and clock gene expression in a mouse model of developmentally primed NASH. Biochim Biophys Acta. 2016;1861(7):584-93.

50. Ren H, Hu F, Wang D, Kang X, Feng X, Zhang L, et al. SIRT2 Prevents Liver Steatosis and Metabolic Disorders via Deacetylation of HNF4alpha. Hepatology. 2021.

51. Xie Z, Li H, Wang K, Lin J, Wang Q, Zhao G, et al. Analysis of transcriptome and metabolome profiles alterations in fatty liver induced by high-fat diet in rat. Metabolism. 2010;59(4):554-60.

52. DeLorenzo RJ, Ruddle FH. Glutamate oxalate transaminase (GOT) genetics in Mus musculus: linkage, polymorphism, and phenotypes of the Got-2 and Got-1 loci. Biochem Genet. 1970;4(2):259-73.

53. Schiele F, Artur Y, Varasteh A, Wellman M, Siest G. Serum mitochondrial aspartate aminotransferase activity: not useful as a marker of excessive alcohol consumption in an unselected population. Clin Chem. 1989:35(6):926-30.

54. Sookoian S, Castano GO, Scian R, Fernandez Gianotti T, Dopazo H, Rohr $C$, et al. Serum aminotransferases in nonalcoholic fatty liver disease are a signature of liver metabolic perturbations at the amino acid and Krebs cycle level. Am J Clin Nutr. 2016;103(2):422-34.

55. Dong Q, Kuefner MS, Deng X, Bridges D, Park EA, Elam MB, et al. Sex-specific differences in hepatic steatosis in obese spontaneously hypertensive (SHROB) rats. Biol Sex Differ. 2018;9(1):40.

56. Xue B, Yang Z, Wang X, Shi H. Omega-3 polyunsaturated fatty acids antagonize macrophage inflammation via activation of AMPK/RIRT pathway. PLoS One. 2012;7(10):e45990.

57. Hodson L, Bhatia L, Scorletti E, Smith DE, Jackson NC, Shojaee-Moradie $F$, et al. Docosahexaenoic acid enrichment in NAFLD is associated with improvements in hepatic metabolism and hepatic insulin sensitivity: a pilot study. Eur J Clin Nutr. 2017;71(8):973-9.

58. Popeijus HE, van Otterdijk SD, van der Krieken SE, Konings M, Serbonij K, Plat J, et al. Fatty acid chain length and saturation influences PPARalpha transcriptional activation and repression in HepG2 cells. Mol Nutr Food Res. 2014:58(12):2342-9.

59. Chen VL, Du X, Chen Y, Kuppa A, Handelman SK, Vohnoutka RB, et al. Genome-wide association study of serum liver enzymes implicates diverse metabolic and liver pathology. Nat Commun. 2021;12(1):816.

60. Karere GM, Glenn JP, VandeBerg JL, Cox LA. Differential microRNA response to a high-cholesterol, high-fat diet in livers of low and high LDL-C baboons. BMC Genomics. 2012;13:320. 
61. Spradling KD, Glenn JP, Garcia R, Shade RE, Cox LA. The baboon kidney transcriptome: analysis of transcript sequence, splice variants, and abundance. PLoS One. 2013;8(4):e57563.

62. Xing Y, Yu T, Wu YN, Roy M, Kim J, Lee C. An expectation-maximization algorithm for probabilistic reconstructions of full-length isoforms from splice graphs. Nucleic Acids Res. 2006;34(10):3150-60.

63. Puppala S, Li C, Glenn JP, Saxena R, Gawrieh S, Quinn A, et al. Primate fetal hepatic responses to maternal obesity: epigenetic signalling pathways and lipid accumulation. J Physiol. 2018;596(23):5823-37.

64. Friedlander MR, Chen W, Adamidi C, Maaskola J, Einspanier R, Knespel S, et al. Discovering microRNAs from deep sequencing data using miRDeep. Nat Biotechnol. 2008;26(4):407-15.

65. Oliveros JC. Venny. An interactive tool for comparing lists with Venn's diagrams. 2015 [Available from: https://bioinfogp.cnb.csic.es/tools/venny/ index.html.

66. Bao Y, Zhao T, Wang X, Qiu Y, Su M, Jia W, et al. Metabonomic variations in the drug-treated type 2 diabetes mellitus patients and healthy volunteers. J Proteome Res. 2009;8(4):1623-30.

67. Qiu Y, Cai G, Su M, Chen T, Zheng X, Xu Y, et al. Serum metabolite profiling of human colorectal cancer using GC-TOFMS and UPLC-QTOFMS.J Proteome Res. 2009;8(10):4844-50.

68. Jonsson P, Johansson Al, Gullberg J, Trygg J, A J, Grung B, et al. Highthroughput data analysis for detecting and identifying differences between samples in GC/MS-based metabolomic analyses. Anal Chem. 2005;77(17):5635-42.

69. Jonsson P, Gullberg J, Nordstrom A, Kusano M, Kowalczyk M, Sjostrom M, et al. A strategy for identifying differences in large series of metabolomic samples analyzed by GC/MS. Anal Chem. 2004;76(6):1738-45.

70. Lisec J, Schauer N, Kopka J, Willmitzer L, Fernie AR. Gas chromatography mass spectrometry-based metabolite profiling in plants. Nat Protoc. 2006;1 (1):387-96

71. Winnike JH, Wei X, Knagge KJ, Colman SD, Gregory SG, Zhang X. Comparison of GC-MS and GCXGC-MS in the analysis of human serum samples for biomarker discovery. J Proteome Res. 2015;14(4):1810-7.

72. Kopka J, Schauer N, Krueger S, Birkemeyer C, Usadel B, Bergmuller E, et al. GMD@CSB.DB: the Golm Metabolome Database. Bioinformatics. 2005;21(8):1635-8.

73. Fordahl S, Cooney P, Qiu Y, Xie G, Jia W, Erikson KM. Waterborne manganese exposure alters plasma, brain, and liver metabolites accompanied by changes in stereotypic behaviors. Neurotoxicol Teratol. 2012;34(1):27-36.

74. Buniello A, MacArthur JAL, Cerezo M, Harris LW, Hayhurst J, Malangone $C$, et al. The NHGRI-EBI GWAS Catalog of published genome-wide association studies, targeted arrays and summary statistics 2019. Nucleic Acids Res. 2019;47(D1):D1005-D12.

\section{Publisher's Note}

Springer Nature remains neutral with regard to jurisdictional claims in published maps and institutional affiliations.

Ready to submit your research? Choose BMC and benefit from:

- fast, convenient online submission

- thorough peer review by experienced researchers in your field

- rapid publication on acceptance

- support for research data, including large and complex data types

- gold Open Access which fosters wider collaboration and increased citations

- maximum visibility for your research: over 100M website views per year

At BMC, research is always in progress.

Learn more biomedcentral.com/submissions 